\title{
Blood meal induced regulation of the chemosensory gene repertoire in the southern house mosquito
}

\author{
Tanvi Taparia ${ }^{1,2}$, Rickard Ignell ${ }^{1 \dagger}$ and Sharon Rose Hill ${ }^{1{ }^{*+}}$
}

\begin{abstract}
Background: The southern house mosquito, Culex quinquefasciatus, is one of the most prevalent vectors of lymphatic filariasis and flavivirus-induced encephalitis. Its vectorial capacity is directly affected by its reproductive feeding behaviors, such as host seeking, blood feeding, resting, and egg laying. In mosquitoes, these gonotrophic behaviors are odor-mediated and regulated following blood feeding. Immediately after a blood meal, female mosquitoes show reduced olfactory responsiveness and flight activity, as they enter a resting state. Insights into antennal chemosensory gene regulation at this time period can provide a foundation to identify targets involved in the state switch between host seeking and resting.

Results: This study used quantitative gene expression analyses to explore blood meal induced regulation of chemosensory gene families in the antennae of 6 days post-emergence C. quinquefasciatus females. Improved annotations for multiple chemosensory gene families, and a quantitative differential gene expression analysis between host seeking and $24 \mathrm{~h}$ post- blood fed females of the same age, allowed for the detection of transcripts that potentially play a role in the switch from host seeking to resting, in C. quinquefasciatus. The expression profiles of chemosensory genes varied significantly between the two treatments.

Conclusions: Annotations for chemosensory gene repertoires in C. quinquefasciatus have been manually curated and corrected for $3^{\prime}$ exon choice and transcript length, through sequence and transcriptome analyses. The gene expression analyses identified various molecular components of the peripheral olfactory system in C. quinquefasciatus, including odorant receptors, ionotropic receptors, odorant binding proteins and chemosensory proteins, that are regulated in response to blood feeding, and could be critical for the behavioral switch from host seeking to resting. Functional characterization of these proteins in the future can identify targets essential for the females' gonotrophic behaviors, and can be used to design novel vector control strategies.
\end{abstract}

Keywords: Culex quinquefasciatus, Chemoreceptors, Chemosensory proteins, Host seeking, Blood meal, Resting, Transcriptome, Olfaction

\section{Background}

Females of the southern house mosquito, Culex quinquefasciatus, require a blood meal to complete each gonotrophic cycle, comprised of alternately host-seeking, blood feeding, resting, and egg laying [1]. This successive reproductive feeding behavior puts more than one fifth of the world's population at risk of vector borne diseases

\footnotetext{
* Correspondence: sharon.hill@slu.se

'Equal contributors

'Unit of Chemical Ecology, Department of Plant Protection Biology, Swedish

University of Agricultural Sciences, Alnarp, Sweden

Full list of author information is available at the end of the article
}

such as lymphatic filariasis and flavivirus-induced encephalitis, including West Nile fever [2]. Similar to other mosquito species, C. quinquefasciatus relies heavily on olfaction to mediate gonotrophic behaviors [3, 4]. Characterization of the gene expression that underpins the switching between the stereotypic odor-mediated behaviors may identify targets that can be used for the development of novel monitoring and control strategies.

Behavioral studies indicate that C. quinquefasciatus are attracted to birds, and bird-derived odorants, as well as human emanates during host seeking $[5,6]$. The identity of these volatiles is only now being described, with 
the sole known attractant to date being nonanal [7]. In addition, some volatiles driving the oviposition behavior of C. quinquefasciatus, ca. 72 hours post-blood meal, have been identified from the preferred egg laying substrate, fermenting vegetation, including for example 4-methylphenol, 3-methylindole, indole, and nonanal [8]. Physiological recordings from the sensilla on the main olfactory organ, the antenna, have shown that olfactory receptor neurons (ORNs) of C. quinquefasciatus and other mosquitoes are tuned to these as well as other odors present in host and oviposition site emissions [9-14].

In insects, odors are detected by the molecular components of the peripheral olfactory system [15]. The lymph soluble proteins, odorant binding proteins (OBPs) and chemosensory proteins (CSPs), are believed to be involved in salient odorant recognition and translocation of volatile chemicals to the ORN dendritic membrane. At the membrane, odorant receptors (ORs), ionotropic receptors (IRs), gustatory receptors (GRs), and sensory neuron membrane proteins (SNMPs) are involved in odorant recognition and transduction of volatiles into electrical signals [16]. Finally, odorant degrading enzymes (ODEs) have been proposed to mediate the subsequent volatile clearance from the lymph [16]. While the majority of members from each of these gene families have been identified in the yellow fever mosquito, Aedes aegypti [17], and the malaria mosquito, Anopheles gambiae $[18,19]$, the annotation of these genes is ongoing in C. quinquefasciatus [20-22]. The genome-wide duplication events in C. quinquefasciatus [21], coupled with the rapid expansion of the chemosensory gene families in insects $[15,23]$, has hitherto constrained the annotation process.

Transcriptome analyses across the antennae of Ae. aegypti and An. gambiae reveal a transient change in expression of chemosensory genes during the first gonotrophic cycle, evident already $24 \mathrm{~h}$ post-blood meal [17, $24,25]$. In general, an overall reduction in chemosensory gene transcripts in the antennae of both Ae. aegypti and An. gambiae is observed following a blood meal, with only a few olfactory receptors being significantly upregulated $[17,24]$. A comparison between Ae. aegypti and $A n$. gambiae indicates that the regulation of chemoreceptor expression post-blood meal is species specific among putative homologues [17, 24]. Moreover, of the five OBPs up-regulated $24 \mathrm{~h}$ post-blood meal ( $>3$ fold) in $A n$. gambiae, three show species specific regulation, while the up-regulation of $A g O B P 9$ and $A g O B P 54$ is reflected in their Ae. aegypti putative homologues [17, 24]. The observed regulation of select chemosensory genes following a blood meal coincides with the olfactory refractoriness in the host-seeking behavior [26-28], reduced flight activity [29], and the change in electrophysiological responses of olfactory sensilla to volatiles [10, 13], in recently blood fed mosquitoes. This endogenous regulation from host seeking to resting behavior is most pronounced $24 \mathrm{~h}$ post-blood meal [4, 10, 13, 24-28, 30, 31]. The differential expression of the associated molecular machinery may provide insights for further investigations into the link between gene expression and olfactory behavior [24].

In this study, we employ high throughput quantitative RNA sequencing to further the existing annotation of chemosensory genes and subsequently to investigate the influence of a blood meal on various chemosensory gene families in C. quinquefasciatus females, $24 \mathrm{~h}$ post-blood feeding, the quiescent period prior to engaging in preoviposition behavior. Key chemosensory genes within the olfactory apparatus are identified that are modulated between host seeking and blood fed females. The potential biological significance of this genetic regulation is discussed in the context of the behavioral switch between host seeking and resting in this anautogenous mosquito.

\section{Methods}

\section{Rearing and tissue collection}

Culex quinquefasciatus (Thai strain) were reared under normal laboratory culture conditions $\left(27 \pm 2{ }^{\circ} \mathrm{C} ; 70 \pm 2 \%\right.$ relative humidity; $12 \mathrm{~h}: 12 \mathrm{~h}$ light-dark photoperiod) [32]. Antennae from cold-anaesthetized reproductively mature adult females, 6 days post-emergence, were dissected directly into RNAlater ${ }^{\circledR}$ (Thermo Fisher Scientific, Sweden). To minimize the effect of the circadian rhythm associated with gene expression, the tissue was collected during the peak of host seeking in early scotophase, zeitgeber time (ZT) $15 \pm 2 \mathrm{~h}$ [33], over multiple days. Collected tissue was stored at $8{ }^{\circ} \mathrm{C}$ overnight and then transferred to $-80{ }^{\circ} \mathrm{C}$ until RNA extraction. While all mosquitoes were provided with ad libitum access to a $10 \%$ sucrose solution, only half of the adult females were provided with an opportunity to blood feed $24 \pm 2 \mathrm{~h}$ prior to dissection. Females were offered sheep blood (Håtuna Lab, Bro, Sweden) from an artificial feeder (Hemotek Discovery Workshops, Accrington, UK). In total, 800 pairs of antennae were collected from females of each cohort, non-blood fed (nbf) and blood fed to completion (bf).

\section{RNA extraction and sequencing}

Tissues were homogenized using a Vibra-Cell sonicator (VCX-130, Sonics and Materials, Newtown, CT) for 10 cycles at $70 \%$ amplitude, $1 \mathrm{~s}$ on and off pulses, repeated three times, interspersed with $30 \mathrm{~s}$ incubations on ice. Total RNA was extracted using an RNeasy Mini Kit (Qiagen, Sweden) according to the manufacturer's protocol, including the on-column RNase-free DNase I 
treatment (Qiagen, Sweden). The RNA was quantified fluorometrically (Qubit, Life Technologies, Sweden) and then stored at $-80{ }^{\circ} \mathrm{C}$. An aliquot of RNA was shipped on dry ice to Eurofins Genomics (Munich, Germany) for library construction and single-end Illumina sequencing (3' RNA-Seq) of the cDNA libraries (Illumina HiSeq 2500). Oligo-dT-based priming of total RNA to extract mRNA was used to generate cDNA libraries. Each library was divided into two technical replicates, which were run on separate channels.

\section{Read mapping and gene annotations}

Prior to mapping, adapter sequences were removed from the raw reads and low quality bases (Phred score $\leq 20)$ from the start and end of each single read were clipped in a sliding window approach (window size $4 \mathrm{bp}$, minimum quality 20) using Trimmomatic 0.20 (http://www.usadellab.org). Reads shorter than a length threshold of $40 \mathrm{bp}$ were also removed. Alignment of filtered reads to the reference-annotated genome forms the basis of RNA-Seq analysis [34]. CLC Genomics Workbench 9.0 (http://www.clcbio.com; Qiagen, DK) was used to map the sequenced reads to the reference genome in VectorBase (CPipJ2.2). While $95.6 \%$ of the total counted fragments mapped to the scaffolds from CPipJ2.2, the majority (55.1\%) aligned to the predicted intergenic regions of the genome. This relatively low mapping frequency $(40.5 \%)$ to the genic regions demonstrates that the latest genome annotation (CPipJ2.2) is still largely a prediction, which is unverified by transcriptome analysis, and is therefore unpredictable in both exon choice and transcript length. Hence, in this study the annotations for chemosensory genes were manually curated from the CPipJ 2.2 supercontigs. Supported by the alignment of reads to scaffolds and sequence analyses, exon-intron boundaries and 3' untranslated regions were modified for 158 chemosensory genes in total (Additional files 1, 2 and 3).

\section{RNA-Seq analysis}

RNA-Seq analyses were performed using CLC Genomics Workbench 9.0 (https://www.qiagenbioinformatics.com/). Read count means, normalized between libraries, were chosen to quantify transcript abundance. The RNA-Seq libraries produced single-end reads from the 3' end of each transcript present, providing a strong correlation between the read count and transcript abundance. A rigorous threshold of less than 10 mean counts across either replicate filtered out transcripts below a background level of abundance from the dataset, similar to the $<1$ RPKM filter that is commonly employed to reduce noise [24]. Genes with mean reads counts above this threshold were considered to be reliably expressed. Quantile-based normalization [35] was performed on each library to increase the detection sensitivity of differential expression for low abundance transcripts. The Kal's weighted $\mathrm{Z}$ test [36], which relies on an approximation of the binomial distribution, was applied on the normalized read counts. It was controlled for false discovery rate (FDR) by applying the Benjamini-Hochberg correction [37]. This analysis generated weighted fold changes (FC) and FDR corrected $P$ values that were used to detect differential expression. Genes that exhibited a FC $>2$ and $P<0.05$ were considered to demonstrate significant differential expression, while those with $1.5 \geq \mathrm{FC} \geq 2$ and $P<0.05$ were considered not to be significantly different, but of potential interest. Normalized expression means were transformed with the addition of a constant (i.e. 1.1) to prevent values of infinitive fold change when one of the two libraries being compared exhibited no basal expression. For visualization, the mean counts were then $\log _{2}$ transformed to clearly distinguish genes of varying levels of abundance.

\section{Quantitative real time PCR}

Quantitative real time polymerase chain reaction (qPCR) analysis was performed on select genes to verify the fold changes demonstrated by the transcriptome. Forward and reverse primer pairs (Additional file 4) were designed using Primer 3 (http://bioinfo.ut.ee/primer30.4.0/primer3/), with the following parameters: oligo size of 20-22 bp, melting temperature of $\sim 60{ }^{\circ} \mathrm{C}, \mathrm{GC} \%$ content of $40-60 \%, 2$ bp GC clamp at 3' end, amplicon size between $80-150 \mathrm{bp}$, and preferably overlapping an intron/exon boundary. cDNA libraries for qPCR analyses were prepared from total RNA (extracted as above), based on Oligo(dT) priming using SuperScript III FirstStrand Synthesis System for RT-PCR (Thermo Fischer Scientific, CA, USA), according to the manufacturer's protocol. Four biological replicate cDNA libraries were generated for each bf and nbf cohort. The cDNA was stored at $-20{ }^{\circ} \mathrm{C}$ until needed. Quantitative PCR was performed using the Platinum SYBR Green qPCR SuperMix-UDG w/ROX (Bio-Rad Laboratories, CA, United States), according to manufacturers' protocol, with minor modifications for optimization. The reactions were carried out in $25 \mu \mathrm{l}$ total volume, containing $12.5 \mu \mathrm{l}$ of Bio-Rad Supermix, $0.5 \mu \mathrm{l}$ of forward and reverse primers (Eurofins Genomics, Munich, Germany), $1 \mu \mathrm{l}$ of cDNA and the remaining volume of DNAseRNAse free, PCR-grade water. Amplification was performed on a BioRad CFX 96 (Bio-Rad Laboratories, CA, United States), with the following program: 2 min cycle at $50{ }^{\circ} \mathrm{C}$, and $2 \mathrm{~min}$ at $95{ }^{\circ} \mathrm{C}$, followed by 40 cycles of $30 \mathrm{~s}$ each at 95,58 and $72{ }^{\circ} \mathrm{C}$. Fluorescence readings were taken for each cycle during the elongation step at $72{ }^{\circ} \mathrm{C}$. Melting curve analyses $\left(65{ }^{\circ} \mathrm{C}\right.$ to $94{ }^{\circ} \mathrm{C}$ in $0.5{ }^{\circ} \mathrm{C}$ steps) were performed after the $40^{\text {th }}$ cycle, to test the specificity of the product amplification. For each plate, a 
water control and negative cDNA synthesis control were included. Each primer pair was tested on three technical replicates for each biological replicate for both bf and nbf cohorts. Gene expression levels were determined using the $\Delta \Delta \mathrm{Cq}$ method [38]. Transcription levels per sample were normalized to a reference factor comprising the geometric means of the three most stable reference genes, Elfa1 (CPIJ016188), Orco (CPIJ009573) and RpS4 (CPIJ003013), and expressed relative to the mean of the control group (nbf) females. Gene expression levels were compared between genes per group (nbf and bf) using a two-tailed paired Student's t-test following verification for normality and homogeneity of residuals using D'Agostino-Pearson test. Statistical significance was adjusted for multiple comparisons.

\section{Results and Discussion}

\section{RNA sequencing data}

Quantitative single-end sequencing of antennal mRNA from nbf and bf cohorts generated a combined sequencing depth of over 71 million cleaned reads (Additional file 5), which is similar to previous studies on related species that have produced total reads in the range of 65 to 240 million [24, 39-41]. Since the C. quinquefasciatus genome contains multiple gene family expansions, including olfactory and gustatory receptors [21], high sequencing depth generates sufficient coverage to detect transcripts with low abundance, e.g., the chemoreceptor gene families $[24,42]$ that are the primary interest of this study. To validate the sufficiency of the coverage, the core eukaryotic genes mapping approach (Cegma) provides a set of reliable gene annotations, whose proportion in the genome assembly provides an estimate of the proportion of all known genes that are present [43]. Cegma genes are predominantly housekeeping genes that code for a group of highly conserved eukaryotic ubiquitous proteins [44]. Of the 358 Cegma genes (Additional file 6) identified in C. quinquefasciatus, transcripts for 331 were detected in the antennal libraries, indicating good sequence coverage of the transcriptome.

Quantile-based between-sample normalization was performed to correct for any read distribution bias amongst the libraries, thereby increasing the accuracy of quantitative comparisons among low expression transcripts [45]. This allowed for an increased sensitivity in detection of differentially expressed genes. The squared Pearson coefficient $\left(r^{2}\right)$ [46] of the between-replicate analyses for all four libraries approached one, implying close agreement between the technical replicates (Additional file 5). The increased coefficient following the normalization of the read counts reiterated the effectiveness of this normalization procedure.

\section{Overall expression profile}

Single-end quantitative RNA-Seq detected a total of 15,187 transcripts in the antennal tissue of C. quinquefasciatus females. Out of these, 7,797 transcripts were reliably expressed in numbers above the rigorous cut-off used to control for background levels of abundance (Additional file 7). The majority of the Cegma genes were reliably expressed in the olfactory tissue, where, out of the 331 Cegma genes detected in C. quinquefasciatus, 290 were present above background abundance (Additional file 6).

A level three gene ontology (GO) analysis of the molecular function was performed to gain an overview of the antennal gene expression, and to observe differences in the proportional representation of the genes involved in various molecular functions between transcripts reliably expressed in the olfactory tissue and transcripts differentially expressed between the nbf and bf cohorts (Fig. 1). Transcripts assigned 'odorant-binding' functions, and which include the majority of the chemosensory genes, represent $5.08 \%$ of the overall number of genes expressed in the antennae, and represent $7.06 \%$ of
A Overall expression

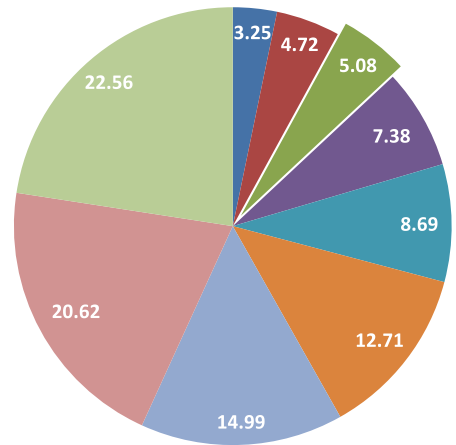

B Differential expression
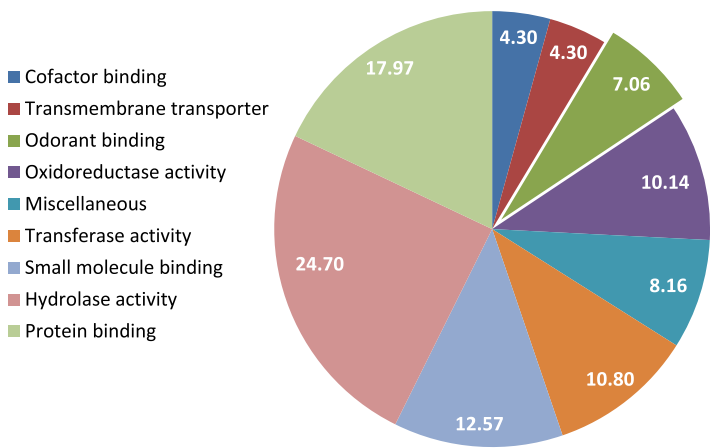

Fig. 1 Level three gene ontology analysis of molecular functions in the antennae of female Culex quinquefasciatus. The total number of genes in the antennal transcriptome that are $\mathbf{a}$ reliably expressed overall and $\mathbf{b}$ differentially expressed between non-blood fed and blood fed cohorts 
the genes which are differentially expressed in the nbf and bf antennae (Fig. 1), indicating that the regulation of genes with an odorant binding function are likely of importance in the behavioral switch between nbf and bf states.

Comparison between the transcriptomes of nbf and bf females yielded 714 genes that were significantly and differentially expressed (Kal's $P<0.05$ and FC $>2$ ) in the antennal tissue (Additional file 7 ). The highest up- or down-regulation of gene expression post-blood feeding was observed for transcripts of various protein degrading enzymes (cathepsins), salivary proteins and yolk proteins (vitellogenins), similar to that observed in the An. gambiae antennal transcriptomes [24]. Two circadian genes, timeless and clock, were significantly down-regulated in the bf cohort, as was also observed in An. gambiae [24]. The majority of the reliably expressed Cegma genes (94.5\%) in the olfactory tissue were not regulated in response to blood feeding, as expected (Additional file 6).

The highly expanded olfactory gene repertoire of $C$. quinquefasciatus $[21,40]$ is thought to reflect the opportunistic feeding behavior of Culex females on birds, humans and livestock, and the diversity observed in oviposition site choice [47]. Out of a repertoire of 180 putative ORs identified in C. quinquefasciatus [21], transcripts for 132 (73\%) were detected in the antennae of 6 days post-emergence (dpe) females, consistent with a previous report [40], of which 96 ORs were reliably expressed above background abundance. This accords with the 56 (70\%) AgORs and 66 (66\%) AaORs detected in $A n$. gambiae and Ae. aegypti, respectively $[17,24]$. This expression correlates with the "one ORto-one ORN class-to-one glomerulus" hypothesis when compared with the expected 50-60 glomeruli in the antennal lobe predicted by comparison with related $\mathrm{Culi-}$ cine species [12, 32, 48].

The IR gene family in C. quinquefasciatus, includes 73 members, of which transcripts for 62 IRs were detected in the antennae of 6 dpe females, consistent with a previous report [40], 30 of which were reliably expressed. This is in line with that reported for $A n$. gambiae (23 out of 46) [19]. Expression for only one of the three co-receptors [49], IR $8 a$, was detected above background abundance, as described previously [40]. Six members of the GR gene family were also expressed in the antennae of $C$. quinquefasciatus, similar in number to that in An. gambiae (13) and Ae. aegypti $(6)[17,24]$. Both culicines show expression for the $\mathrm{CO}_{2}$-sensitive GR gene, GR2, as well as each expressing a single sugar-sensitive GR gene, CqGR8 and AaGr6 [24], whereas neither of these subfamily GR genes are shown to be expressed in the antennae of An. gambiae [24].
Out of a repertoire of 109 OBPs [50], transcripts for 74. OBPs were detected in the antennae, 63 of which were reliably expressed (58\%), following a significant improvement in annotation compared to previous reports [40]. This is in line with that reported for An. gambiae (61\%), while the expression of OBP genes in the antennae of Ae. aegypti (39\%) is lower [17, 24]. The CSP gene family comprises 27 members in C. quinquefasciatus [40], out of which transcripts for 19 CSPS were detected in the antennae of females 6 dpe, with reliable expression for 12. In most mosquito species, the SNMP family comprises two members [51], SNMP1 and SNMP2, whereas in Culex, the family has expanded to comprise four members, SNMP1a, 1b, 1c and SNMP2 [40]. Reliable expression above background abundance was observed for only two SNMPS in the antennal tissue.

\section{Regulation of olfactory genes}

For the majority of the chemosensory genes, an overall lower level of transcription was observed in the female antennae of bf $\left(3.6 \times 10^{6}\right.$ reads $)$ compared to $\mathrm{nbf}\left(3.8 \times 10^{6}\right.$ reads) mosquitoes. This differs from the change in overall level of transcription, which is found to be higher in the bf $\left(14.7 \times 10^{6}\right.$ reads $)$ compared to the nbf $\left(12.8 \times 10^{6}\right.$ reads $)$ mosquitoes (Additional file 7 ). This highlights the importance of the chemosensory function of the antennae during host seeking, and is in accordance with studies on $A e$. aegypti and An. gambiae [17, 24]. For these species, a reduction in olfactory responsiveness towards host cues post-blood feeding has been well documented, behaviorally and physiologically [10, 13, 27, 28, 52]. Following a blood meal, the olfactory responsiveness of female mosquitoes to host cues gradually decreases until females are fully refractory to host odors, $24 \mathrm{~h}$ after a complete blood meal. By this time mosquitoes have located a resting site where females remain quiescent until the preoviposition behavior begins between $48 \mathrm{~h}$ and $96 \mathrm{~h}$ post-blood meal $[28,53]$.

Aedes aegypti females, $24 \mathrm{~h}$ post-blood feeding, are known to down-regulate their olfactory sensitivity towards lactic acid, a host cue [31], and up-regulate their sensitivity towards oviposition cues, such as indoles and phenols [13]. Similarly, in An. gambiae, down- and upregulation of ORN sensitivity to host and oviposition cues, respectively, have been observed post-blood feeding [14], coinciding with the selective regulation of OBPs, ORs and IRs [24]. These changes are believed to modulate the chemosensory apparatus to inhibit the host-seeking response during the resting state and later to revive the system for subsequent gonotrophic events, including the pre-oviposition behavior [10, 13, 24]. It is noteworthy that the chemosensory system of Ae. aegypti begins to reconfigure its sensitivity towards oviposition site cues as early as $24 \mathrm{~h}$ post-blood meal [13]. 


\section{Odorant receptors}

While the overall abundance of the tuning ORs in the antennae was reduced by one fifth in bf compared to nbf females, the obligate co-receptor, ORco, which had the highest transcript abundance in both cohorts, remained unregulated (Fig. 2a). This is in accordance with that of $A g O R c o$ [24] and AaORco [17].The invariant transcript levels of mosquito ORco post-blood meal, as opposed to that of the obligate blood feeder Rhodnius prolixus [54], suggest different roles of the co-receptor in regulating the observed plasticity in olfactory responsiveness among hematophagous insects.

Changes in abundance were observed for the tuning CqORs, with $16 \mathrm{CqORs}$ regulated in response to blood feeding (Fig. 2a). CqORs having higher transcript abundance in nbf females included OR64 and OR53 (listed in ascending magnitude of fold change) with $\mathrm{FC}>2$ and $P<0.05$, and $O R 22$ and $O R 1$ with $\mathrm{FC}>2$ and $P>0.05$. CqORs having higher transcript abundance in bf females included OR54 with $\mathrm{FC}>2$ and $P<0.05$, and $O R 77$ with $\mathrm{FC}>2$ and $P>0.05$. Another ten CqORs of interest $(1.5<\mathrm{FC}<2)$ included OR99, OR160, OR108, OR27, OR174, OR73, OR170, OR150, OR120 that had higher abundance in nbf females, and OR111, which had a higher abundance in bf females. The gene expression profiles of six of the differentially expressed OR genes determined by transcriptome analyses (OR27, OR53, OR64, OR73, OR150, and OR170), were confirmed using qPCR analyses (Additional file 8).

The ligand tuning of most CqORs has not been determined, and therefore a systematic effort to deorphanize these receptors will be required to address the functional relevance of the observed gene expression changes. Of the ORs present in higher abundance in the antennae of host seeking C. quinquefasciatus females (nbf), OR73 is part of a Culex-specific expansion group [21]. OR73 is tuned to multiple phenolic compounds including 4methyl and 4-ethylphenol [55], which are components of human headspace collections [56, 57]. However, as of now there is no direct behavioral evidence documenting the use of phenols by C. quinquefasciatus females as host cues. Moreover, ethyl- and methylphenols are also known oviposition attractants [58]. Down-regulation of OR73, 24 h post-blood feeding, could thus be attributed to either the refractoriness to host cues or the delay of the onset of pre-oviposition behaviors. The downregulated OR1, a receptor tuned to multiple host volatiles, including 1-hexanol, 1-octen-3-ol, 2phenoxyethanol, and benzaldehyde [55], illustrates that ORs tuned to host volatiles are pivotal in regulating the behavioral switch from host seeking to resting.

\section{Ionotropic receptors}

The ionotropic co-receptor, IR8a, was the most abundant transcript, with invariant levels between the bf and nbf cohorts (Fig. 2b), consistent with that found in $A n$. gambiae [24] and Ae aegypti [17], but opposed to $R$. prolixus [54]. Thus, the regulation of chemosensory coreceptors, including ORco, in hematophagous insects seems to be a result of convergent evolution and diverse feeding strategies. In contrast, eight CqIRs in the antennal tissue were regulated in response to blood feeding (Fig. 2b). Six of these, IR41a, IR92g, IR75h.1, IR21a.1, IR75e.1, and IR75m.2 were present in higher abundance in the nbf cohort, and two of them, IR64a and IR123

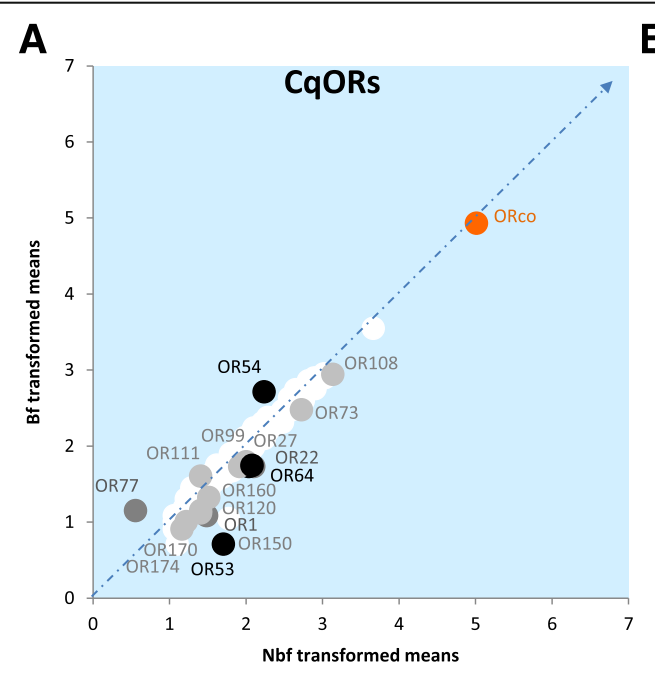

B

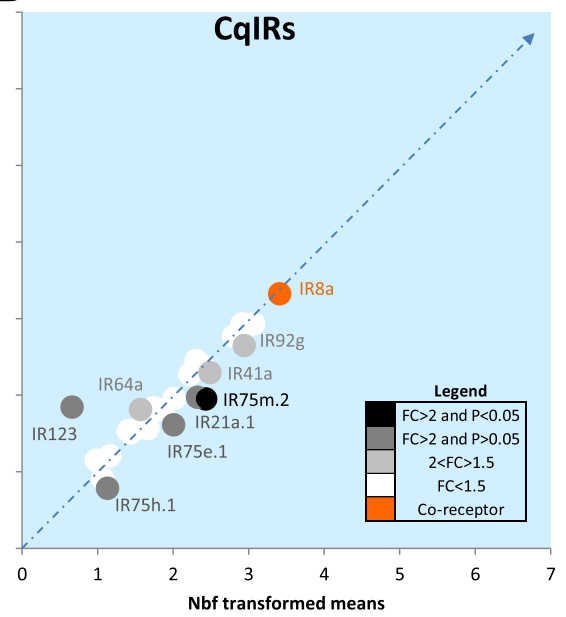

Fig. 2 Differential regulation of chemosensory receptors in Culex quinquefasciatus antennae pre- and post-blood meal. The differential transcript abundance in the antennae of non-blood fed and blood fed females, 6 days post-emergence, are described for $\mathbf{a}$ odorant receptors (ORs) and $\mathbf{b}$ ionotropic receptors (IRs). Transcripts that exhibit significant differences in abundance (Kal's test; $P<0.05$ ), are denoted according to their weighted fold change (FC) 
were present in higher abundance in the bf cohort. The qPCR results (Additional file 8) demonstrated statistically significant differential regulation of six of the IR genes (IR21a, IR64a, IR75e.1, IR75h.1, IR75m.2, and $I R 92 g$ ), thus confirming the expression profiles described by the transcriptomic analyses. The functional characterization of IRs in mosquitoes is only now becoming available [19].

The conserved antennal IR75 subfamily in C. quinquefasciatus comprises 15 members, of which 11 were reliably expressed, and three were down-regulated in response to blood feeding. IRs are involved in the detection of carboxylic acids and amines $[10,19,59,60]$ that are known host and oviposition volatiles, which have a low representation in the odor space of ORs [61, 62]. The IR75 clade is suggested to respond to closely related volatiles [61, 63], and their selective down-regulation post-blood meal indicates their involvement in host selection. Interestingly, IR123 and IR64a were upregulated $24 \mathrm{~h}$ post-blood feeding. In Drosophila, IR64a is responsible for acid avoidance responses [64], and the increased abundance of IR64a in the bf cohort may thus signal aversion to host carboxylic acids following a blood meal.

\section{Odorant binding proteins}

Thirty-four $C q O B P s$ in the antennae were regulated in response to blood feeding (Fig. 3a). Eight $\mathrm{CqOBPs}$ with FC > 2 and $P<0.05$, eight $C q O B P s$ with $\mathrm{FC}>2$ and $P>0.05$, and another eight $C q O B P s$ with $1.5<\mathrm{FC}>2$ were abundant in the nbf cohort. In contrast, four $C q O B P s$ were abundant in the bf cohort with $\mathrm{FC}>2$ and $P<0.05$, five $C q O B P s$ with $\mathrm{FC}>2$ and $P>0.05$ and one, $C q O B P 52$, with FC of 1.67. Little information is available on the functional characterization of $\mathrm{CqOBPs}$, without which few conclusions can be drawn from the expression data alone. Larger numbers of $C q O B P s$ were up-regulated in bf females when compared to other chemosensory gene families. A similar trend was observed in An. gambiae, where most AgOBPs were down-regulated immediately after blood feeding and then up-regulated at $24 \mathrm{~h}$ post-blood meal [24]. This suggests that the olfactory apparatus of females is transiently changing at this early stage in the gonotrophic cycle, likely in response to the physiological and behavioral requirements.

\section{Other chemosensory proteins}

The highest transcript abundance among the CSPS was observed for CSP4 and CSP24, neither of which were regulated in response to blood feeding. Five other CSPs were differentially expressed between the nbf and bf cohorts (Fig. 3b). Transcripts with higher abundance in nbf females included CSP14 and CSP12 with FC $>2$ and $P<0.05$, and CSP 15 and CSP 13 with $1.5<\mathrm{FC}<2$. CSP23 was the only up-regulated CSP in the female antennae $24 \mathrm{~h}$ post - blood meal. Both of the expressed SNMPS were unregulated in response to blood feeding.

\section{Conclusions}

In this study, we have demonstrated blood meal induced regulation of chemosensory genes in the antennae of $C$. quinquefasciatus females. We highlight candidate chemosensory genes that are differentially expressed between nbf and bf females. The functional characterization of these proteins can provide critical insights into the regulation of host seeking and postblood feeding behaviors, and their de-orphanization can

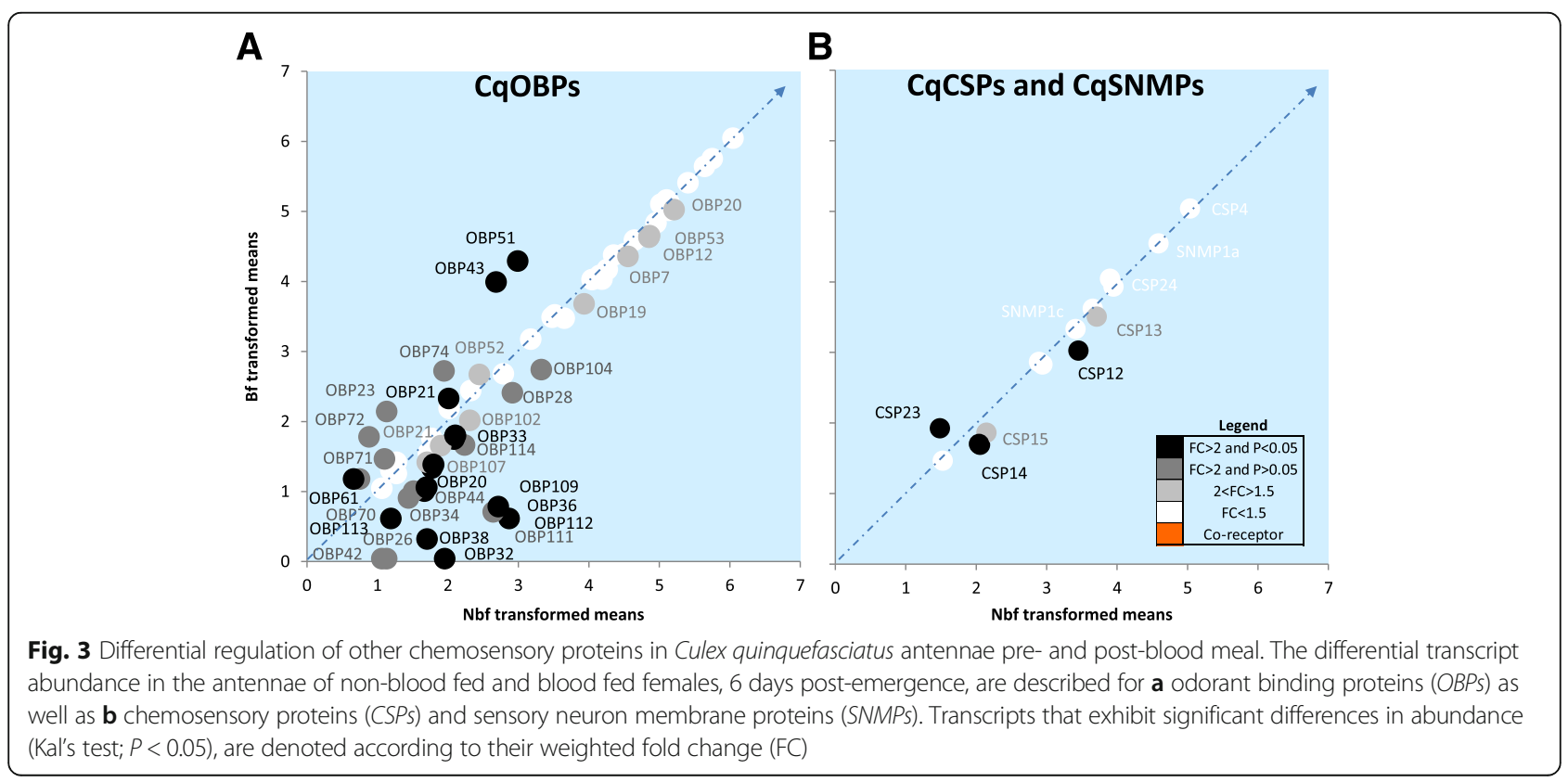


provide a basis for understanding odor coding in female C. quinquefasciatus. As such, this quantitative RNA-Seq analysis provides insights into the genetic regulation of the peripheral olfactory system of C. quinquefasciatus females.

\section{Additional files}

Additional file 1: Lists the names and VectorBase IDs for Culex quinquefasciatus chemosensory gene families. (XLSX $22 \mathrm{~kb}$ )

Additional file 2: Lists the modified mRNA and peptide sequences of the Culex quinquefasciatus odorant receptor gene family. (XLSX $140 \mathrm{~kb}$ )

Additional file 3: Culex quinquefasciatus gene-set with corrected intronexon boundaries and 3' UTRs for 158 chemosensory genes. (TXT $25.6 \mathrm{mb}$ )

Additional file 4: Lists the sequences, amplicon sizes, melting temperatures, GC \% content and 3' complementarity of the forward and reverse primer-pair for each of the reference genes and select ORs and IRs that were verified using qPCR analyses. (DOCX $29 \mathrm{~kb}$ )

Additional file 5: Describes the sequencing depth and Pearson coefficients of the technical replicates from non-blood fed and blood fed libraries. (XLSX $10 \mathrm{~kb}$ )

Additional file 6: Lists the names, read count means and Kal's test statistics for the Cegma genes identified in Culex quinquefasciatus. (XLSX $100 \mathrm{~kb}$ )

Additional file 7: Lists the read count means and Kal's test statistics for all transcripts known to be reliably expressed (present above the cut-off filter). (XLSX 1909 kb)

Additional file 8: Depicts relative gene expression of select IRs and ORs in non-blood fed and blood fed libraries, as determined by real time quantitative polymerase chain reaction. (PDF $86 \mathrm{~kb}$ )

\section{Abbreviations}

bf: Blood fed; bp: base pair; cDNA: complementary deoxyribonucleic acid; Cegma: Common eukaryotic gene mapping approach; CSP: Chemosensory protein; FC: Weighted fold change; FDR: False discovery rate; GC: Guanine and cytosine; GO: Gene ontology; GR: Gustatory receptor; IR: Ionotropic receptor gene; nbf: non-blood fed; OBP: Odorant-binding protein; OR: Odorant receptor; ORco: Obligate OR co-receptor; ORN: Olfactory receptor neuron; qPCR: quantitative real-time polymerase chain reaction; RNA: Ribonucleic acid; RNA-seq: Quantitative mRNA sequencing; RPKM: Reads per kilobase of transcript length per million mapped reads; SNMP: Sensory neuron membrane protein

\section{Acknowledgements}

We thank Kátya Bokán, Thomas Svensson and Anaïs Tallon for their help in tissue collection.

\section{Funding}

This work was funded by the Linnaeus initiative 'Insect Chemical Ecology, Ethology and Evolution' IC-E3 (The Swedish Research Council Formas; Swedish University of Agricultural Sciences). The funding body has had no role in the design of the study and collection, analysis, and interpretation of data, or in writing the manuscript.

\section{Availability of data and materials}

All cleaned reads from each of the four libraries (SRS2163390-SRS2163393) are deposited in the NCBI traces database as SRA study SRP106313 under the BioProject number PRJNA385151 as the following experiments: NBF_2 (SRX2780295), NBF_1 (SRX2780296), BF_2 (SRX2780297) and BF_1 (SRX2780298). Gene set annotations, expression data and sequences of modified genes are available as additional files with this manuscript.

\section{Authors' contributions}

$\mathrm{RI}$ and SRH contributed to the conception and design of this study. TT and $\mathrm{SRH}$ acquired the data. $\Pi$, SRH and RI were involved in the analysis and interpretation of the data. All authors have written and approved the final version of the manuscript.

\section{Competing interests}

The authors declare that they have no competing interests.

\section{Consent for publication}

Not applicable.

\section{Ethics approval}

Mosquitoes used in this study are from our laboratory rearing facility and are Culex quinquefasciatus (Thai strain) originally sourced from the London School of Hygiene \& Tropical Medicine. The blood used for feeding the mosquitoes is defibrinated sheep blood sourced from Håtuna Lab in Bro, Sweden.

\section{Publisher's Note}

Springer Nature remains neutral with regard to jurisdictional claims in published maps and institutional affiliations.

\section{Author details}

${ }^{1}$ Unit of Chemical Ecology, Department of Plant Protection Biology, Swedish University of Agricultural Sciences, Alnarp, Sweden. ${ }^{2}$ Present Address: Department of Environmental Sciences, Wageningen University and Research, Wageningen, The Netherlands.

Received: 12 August 2016 Accepted: 10 May 2017

Published online: 19 May 2017

References

1. Subra R. Biology and control of Culex pipiens quinquefasciatus Say, 1823 (Diptera, Culicidae) with special reference to Africa. Int J Trop Insect Sci. 1981:1:319-38.

2. WHO. Vector-borne diseases. WHO. World Health Organization. 2016.

3. Mclver S. Comparative studies on the sense organs on the antennae and maxillary palps of selected male culicine mosquitoes. Can J Zool. 1971:49:235-9.

4. Davis EE, Bowen MF, Bowen MF. Sensory physiological basis for attraction in mosquitoes. J Am Mosq Control Assoc. 1994;10:316-25.

5. Allan SA, Bernier UR, Kline DL. Laboratory evaluation of avian odors for mosquito (Diptera: Culicidae) attraction. J Med Entomol. 2006:43:225-31.

6. Mboera LEG, Takken W. Odour-mediate host preference of Culex quinquefasciatus in tanzania. Entomol Exp Appl. 1999;92:83-8.

7. Syed Z, Leal WS. Acute olfactory response of Culex mosquitoes to a humanand bird-derived attractant. Proc Natl Acad Sci. 2009;106:18803-8.

8. Afify A, Galizia CG. Chemosensory cues for mosquito oviposition site selection. J Med Entomol. 2015;52:120-30.

9. Bowen MF. Terpene-sensitive receptors in female Culex pipiens mosquitoes: electrophysiology and behaviour. J Insect Physiol. 1992;38:759-64.

10. Davis EE. Development of lactic acid-receptor sensitivity and host-seeking behaviour in newly emerged female Aedes aegypti mosquitoes. J Insect Physiol. 1984;30:211-5.

11. Takken W, Knols BGJ. Odor-mediated behavior of Afrotropical malaria mosquitoes. Annu Rev Entomol. 1999:44:131-57.

12. Ghaninia M, Ignell $R$, Hansson BS. Functional classification and central nervous projections of olfactory receptor neurons housed in antennal trichoid sensilla of female yellow fever mosquitoes, Aedes aegypti. Eur J Neurosci. 2007;26:1611-23.

13. Siju KP, Hill SR, Hansson BS, Ignell R. Influence of blood meal on the responsiveness of olfactory receptor neurons in antennal sensilla trichodea of the yellow fever mosquito, Aedes aegypti. J Insect Physiol. 2010;56:659-65.

14. Qiu YT, van Loon JJA, Takken W, Meijerink J, Smid HM. Olfactory coding in antennal neurons of the malaria mosquito, Anopheles gambiae. Chem Senses. 2006;31:845-63.

15. Suh E, Bohbot JD, Zwiebel LJ. Peripheral olfactory signaling in insects. Curr Opin Insect Sci. 2014;6:86-92.

16. Leal WS. Odorant reception in insects: roles of receptors, binding proteins, and degrading enzymes. Annu Rev Entomol. 2013:58:373.

17. Matthews BJ, McBride CS, DeGennaro M, Despo O, Vosshall LB. The neurotranscriptome of the Aedes aegypti mosquito. BMC Genomics. 2016;17:32.

18. Hill CA, Fox AN, Pitts RJ, Kent LB, Tan PL, Chrystal MA, et al. G protein coupled receptors in Anopheles gambiae. Science. 2002;298:176-8.

19. Pitts RJ, Derryberry SL, Zhang Z, Zwiebel LJ. Variant ionotropic receptors in the malaria vector mosquito Anopheles gambiae tuned to amines and carboxylic Acids. Sci Rep. 2017;7:40297. 
20. Pelletier J, Leal WS. Genome analysis and expression patterns of odorantbinding proteins from the Southern House mosquito Culex pipiens quinquefasciatus. PLoS One. 2009;4:e6237.

21. Arensburger P, Megy K, Waterhouse RM, Abrudan J, Antelo B, Bartholomay $L$, et al. Sequencing of Culex quinquefasciatus establishes a platform for mosquito comparative genomics. Science. 2013;330:86-8.

22. Pelletier J, Leal WS. Characterization of olfactory genes in the antennae of the Southern house mosquito, Culex quinquefasciatus. J Insect Physiol. 2011; 57:915-29.

23. Sánchez-Gracia A, Vieira FG, Rozas J. Molecular evolution of the major chemosensory gene families in insects. Heredity (Edinb). 2009;103:208-16. Available from: http://dx.doi.org/10.1038/hdy.2009.55.

24. Rinker DC, Pitts RJ, Zhou X, Suh E, Rokas A, Zwiebel LJ. Blood meal-induced changes to antennal transcriptome profiles reveal shifts in odor sensitivities in Anopheles gambiae. Proc Natl Acad Sci. 2013:110:8260-5.

25. Fox AN, Pitts RJ, Robertson HM, Carlson JR, Zwiebel LJ. Candidate odorant receptors from the malaria vector mosquito Anopheles gambiae and evidence of down-regulation in response to blood feeding. Proc Natl Acad Sci. 2001;98:14693-7.

26. Klowden MJ, Lea AO. Humoral inhibition of host-seeknig in Aedes aegypti during oocyte maturation. J Insect Physiol. 1979:25:231-5.

27. Klowden MJ, Lea AO. Abdominal distention terminates subsequent hostseeking behaviour of Aedes aegypti following a blood meal. J Insect Physiol. 1979:25:583-5.

28. Takken W, Van Loon JJA, Adam W. Inhibition of host-seeking response and olfactory responsiveness in Anopheles gambiae following blood feeding. J Insect Physiol. 2001;47:303-10.

29. Jones MDR. The programming of circadian flight-activity in relation to mating and the gonotrophic cycle in the mosquito, Aedes aegypti. Physiol Entomol. 1981;6:307-13.

30. Klowden MJ, Lea AO. Blood meal size as a factor affecting continued hostseeking by Aedes aegypti (L.). Am J Trop Med Hyg. 1978;27:827-31.

31. Davis EE. Regulation of sensitivity in the peripheral chemoreceptor systems for host-seeking behaviour by a haemolymph-borne factor in Aedes aegypti. J Insect Physiol. 1984;30:179-83.

32. Hill SR, Hansson BS, Ignell R. Characterization of antennal trichoid sensilla from female Southern house mosquito, Culex quinquefasciatus say. Chem Senses. 2009;34:231-52.

33. Yee WL, Foster WA. Diel sugar-feeding and host-seeking rhythms in mosquitoes (Diptera: Culicidae) under laboratory conditions. J Med Entomol. 1992;29:784-91.

34. Wang Z, Gerstein M, Snyder M. RNA-Seq: a revolutionary tool for transcriptomics. Nat Rev Genet. 2009;10:57-63.

35. Bolstad BM, Irizarry R, Astrand M, Speed TP. A comparison of normalization methods for high density oligonucleotide array data based on variance and bias. Bioinformatics. 2003;19:185-93.

36. Kal AJ, van Zonneveld AJ, Benes V, van den Berg M, Koerkamp MG, Albermann $\mathrm{K}$, et al. Dynamics of gene expression revealed by comparison of serial analysis of gene expression transcript profiles from yeast grown on two different carbon sources. Mol Biol Cell. 1999;10:1859-72.

37. Benjamini $Y$, Hochberg $Y$. Controlling the false discovery rate: a practical and powerful approach to multiple testing. J R Stat Soc Ser B. 1995;57:289-300.

38. Livak KJ, Schmittgen TD. Analysis of relative gene expression data using realtime quantitative $P C R$ and the $2-\Delta \Delta C T$ method. Methods. 2001;25:402-8.

39. Bonizzoni M, Dunn WA, Campbell CL, Olson KE, Dimon MT, Marinotti O, et al. RNA-seq analyses of blood-induced changes in gene expression in the mosquito vector species, Aedes aegypti. BMC Genomics. 2011;12:82.

40. Leal WS, Choo Y, Xu P, da Silva CSB, Ueira-Vieira C. Differential expression of olfactory genes in the southern house mosquito and insights into unique odorant receptor gene isoforms. Proc Natl Acad Sci. 2013;110:18704-9.

41. McBride CS, Baier F, Omondi AB, Spitzer SA, Lutomiah J, Sang R, et al. Evolution of mosquito preference for humans linked to an odorant receptor. Nature. 2014;13:222-7.

42. Justice RW, Dimitratos S, Walter MF, Woods DF, Biessmann H. Sexual dimorphic expression of putative antennal carrier protein genes in the malaria vector Anopheles gambiae. Insect Mol Biol. 2003;12:581-94.

43. Parra G, Bradnam K, Ning Z, Keane T, Korf I. Assessing the gene space in draft genomes. Nucleic Acids Res. 2009;37:289-97.

44. Parra G, Bradnam K, Korf I. CEGMA: a pipeline to accurately annotate core genes in eukaryotic genomes. Bioinformatics. 2007;23:1061-7.
45. Bullard JH, Purdom E, Hansen KD, Dudoit S. Evaluation of statistical methods for normalization and differential expression in mRNA-Seq experiments. BMC Bioinformatics. 2010;11:94.

46. Pearson, K. On further methods of determining correlation. Mathematical contributions to the theory of evolution. Volume 16. Biometric Series IV. London: Cambridge University Press. p. 39. ISBN: 9785518901315.

47. Takken W, Verhulst NO. Host preferences of blood-feeding mosquitoes. Annu Rev Entomol. 2013;58:433-53.

48. Ignell R, Dekker T, Ghaninia M, Hansson BS. Neuronal architecture of the mosquito deutocerebrum. J Comp Neurol. 2005;493:207-40.

49. Abuin L, Bargeton B, Ulbrich MH, Isacoff EY, Kellenberger S, Benton R. Functional architecture of olfactory ionotropic glutamate receptors. Neuron. 2011;69:44-60.

50. Manoharan M, Chong MNF, Vaitinadapoul A, Frumence E, Sowdhamini R, Offmann B. Comparative genomics of odorant binding proteins in Anopheles gambiae, Aedes aegypti, and Culex quinquefasciatus. Genome Biol Evol. 2013;5:163-80.

51. Vogt RG, Miller NE, Litvack R, Fandino RA, Sparks J, Staples J, et al. The insect SNMP gene family. Insect Biochem Mol Biol. 2009;39:448-56.

52. Klowden MJ. Endogenous regulation of the attraction of Aedes aegypti mosquitoes. J Am Mosq Control Assoc. 1994;10:326-32.

53. Brown MR, Klowden MJ, Crim JW, Young L, Shrouder LA, Lea AO. Endogenous regulation of mosquito host-seeking behavior by a neuropeptide. J Insect Physiol. 1994:40:399-406.

54. Latorre Estivalis JM, Omondi BA, DeSouza O, Oliveira IHR, Ignell R, Lorenzo MG. Molecular basis of peripheral olfactory plasticity in Rhodnius prolixus, a Chagas disease vector. Front Ecol Evol. 2015;3:74

55. Xu P, Choo YM, Pelletier J, Sujimoto FR, Hughes DT, Zhu F, et al. Silent, generic and plant kairomone sensitive odorant receptors from the Southern house mosquito. J Insect Physiol. 2013;59:961-6.

56. Hallem EA, Nicole Fox A, Zwiebel L, Carlson JR. Olfaction: mosquito receptor for human-sweat odorant. Nature. 2004:427:212-3.

57. Curran AM, Rabin SI, Prada PA, Furton KG. Comparison of the volatile organic compounds present in human odor using SPME-GC/MS. J Chem Ecol. 2005:31:1607-19.

58. Millar JG, Chaney JD, Mulla MS. Identification of oviposition attractants for Culex quinquefasciatus from fermented Bermuda grass infusions. J Am Mosq Control Assoc. 1992;8:11-7.

59. van Loon JJA, Smallegange RC, Bukovinszkiné-Kiss G, Jacobs F, De Rijk M, Mukabana WR, et al. Mosquito attraction: crucial role of carbon dioxide in formulation of a five-component blend of human-derived volatiles. J Chem Ecol. 2015;41:567-73.

60. Hussain A, Zhang M, Üçpunar HK, Svensson T, Quillery E, Gompel N, et al. Ionotropic chemosensory receptors mediate the taste and smell of polyamines. PLoS Biol. 2016;14, e1002454.

61. Croset V, Rytz R, Cummins SF, Budd A, Brawand D, Kaessmann H, et al. Ancient protostome origin of chemosensory ionotropic glutamate receptors and the evolution of insect taste and olfaction. PLoS Genet. 2010;6, e1001064.

62. Benton R, Vannice KS, Gomez-Diaz C, Vosshall LB. Variant ionotropic glutamate receptors as chemosensory receptors in Drosophila. Cell. 2009; 136:149-62.

63. Rytz R, Croset V, Benton R. lonotropic Receptors (IRs): chemosensory ionotropic glutamate receptors in Drosophila and beyond. Insect Biochem Mol Biol. 2013:43:888-97.

64. Ai M, Min S, Grosjean Y, Leblanc C, Bell R, Benton R, et al. Acid sensing by the Drosophila olfactory system. Nature. 2010:468:691-5. 